\title{
Performance Analysis and Simulation of UAV Data Link and Communication Link
}

\author{
Junkai Ma ${ }^{\text {a) }}$, Hongbo Liu \\ College of Electronic Engineering, Naval University of Engineering, Wuhan, Hubei, 430033, China \\ a)752968873@qq.com
}

\begin{abstract}
According to the demand of optimization design of UAV communication system, this article proposes a method of calculating the link loss of UAV data chain by studying the channel characteristics of UAV data link; establishes the path loss of UAV channel of UAV Model; analyzes the influence of frequency, receiving antenna gain and space environment upon channel path loss. Simulation and experiment are carried out for channel loss model of UAV in specific areas. The comparison between the measured data and the simulation data shows that this model is effective in UAV link loss calculation, which can provide important theoretical reference for UAV data link communication system design.
\end{abstract}

Key words: UAV data link; channel model; path loss; communication link.

\section{INTRODUCTION}

Unmanned Aerial Vehicle (UAV) is an aircraft that uses autonomous wireless control equipment or an on-board automatic control system to accomplish autonomous flight control. As one of the important components of UAV system, UAV data link, in order to achieve accurate, efficient and reliable data transmission between open spaces, is mainly responsible for data communication between aircraft and ground stations. The efficiency of its performance will directly affect the overall level of the UAV system [1]. UAV data chain demonstration process had found that the UAV data chain moved rapidly with the air platform movement. The arrival of the data link to receive the terminal signal in the propagation process experienced an unpredictable decline. The UAV channel if compared with the general channel showed its unique characteristics, hence increased the complexity in data link communication terminal design and parameter selection. Adjust hardware and software equipment parameter continuously to meet the performance of their communications is usually applied in actual work. Therefore, at the beginning of system design, it is very important to improve stability and economic efficiency of the system by simulating and analyzing the characteristics of channel fading in data link of UAV, which can optimize design process and shorten design cycle.

\section{UAV DATA LINK CHANNEL LINK CHARACTERISTICS}

The performance of UAV data link system is restricted by wireless channels. The working frequency band of UAV data link usually ranges from $150 \mathrm{MHz}$ to $1.5 \mathrm{GHz}$; the communication radius is from 1 to $100 \mathrm{Km}$; and the antenna height is from 10 to $1000 \mathrm{~m}$. UAV data link channel characteristics are shown in Figure 1. When UAV is affected by topography, ground objects and other factors, there will be a strong channel fading phenomenon in UAV's communication channel [2]. 


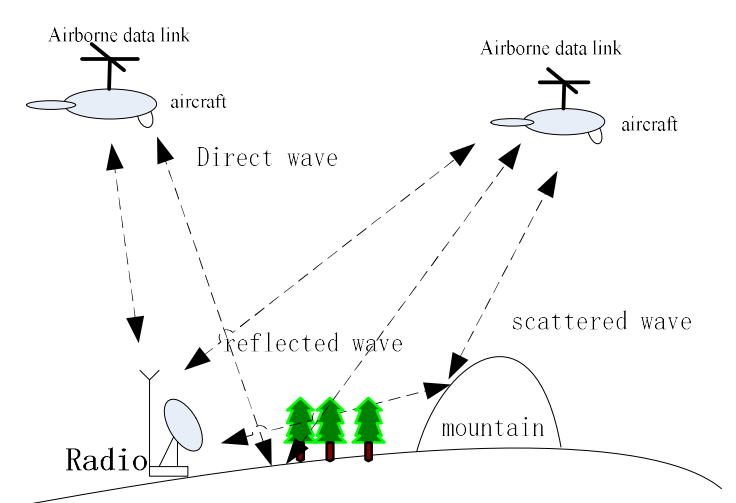

Horizon line

FIGURE.1 UAV data link channel diagram

UAV data link channel path loss affect the overall communication system design, so in order to determine the parameters of the communication system, it is necessary to have accurate path loss calculation method.

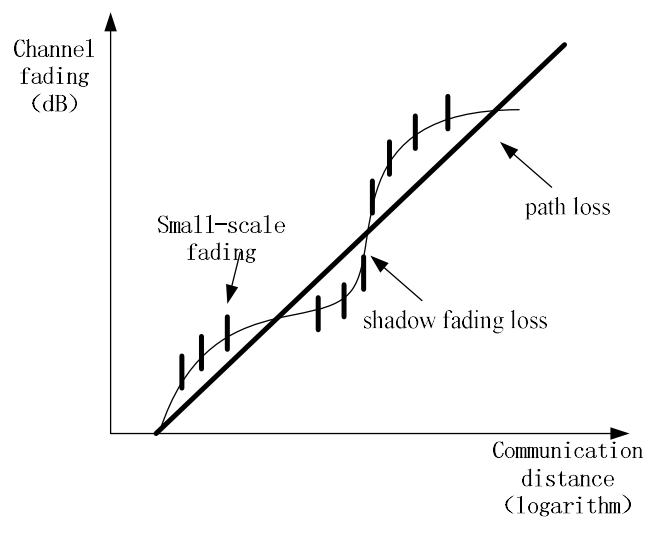

FIGURE .2 UAV data link channel fading diagram

The wireless link between UAVs is affected by the environment. Therefore, the losses in the channel link mainly include propagation path loss, shadow fading loss, frequency selective fading and atmospheric fading [3]. The loss for UAV transmission link channel is mainly path loss. Therefore, this article mainly studies channel characteristic of data link of UAV in terms of path loss and shadow fading loss. UAV data link channel fading composition is shown in Figure 2. According to the basic factors of data link terminal, including the frequency, the receiving and transmitting antenna gain, and the influence of location in space environment on the channel path loss are simulated and analyzed. The conclusion of this research provided a reliable theoretical basis for the design and development of UAV data link communication system in future.

\section{ESTABLISHMENT AND SIMULATION OF DATA LINK COMMUNICATION LINK LOSS MODEL}

\section{Channel path loss model analysis}

UAV data link transmission channel are used for UHF channel. The frequency range: $300 \sim 3000 \mathrm{MHZ}$. The main transmission mode is line of sight (LOS) transmission. At this point, D indicates the distance between the source and the receiver (unit: $\mathrm{m}$ ). When UAV uses different antennas, the gain of the transmitting antenna is Gt and the gain of the receiving antenna is $\mathrm{Gr}$, the power of the received signal at distance D is $\operatorname{Pr}(\mathrm{d})$, the signal path loss [4]: 


$$
\mathrm{P}_{\mathrm{r}}(D)=\frac{P_{t} G_{t} G_{r} C}{(4 \pi)^{2} D^{2} S f_{c}}
$$

$\mathrm{P}_{\mathrm{r}}$ is the launch power (unit: $\mathrm{W}$ ), $C=3.0 \times 108 \mathrm{~m} / \mathrm{s}, \mathrm{S}$ is the system loss coefficient which indicates the total loss of the hardware system in UAV data link system and has nothing to do with the propagation path. Usually $\mathrm{S} \geq 1$, from the above formula (1), power that can be received will decay exponentially with time D.

For the convenience of calculation, the effect of system loss on channel loss will be ignored. Therefore, it is assumed that the free-space path loss PSF (D) is:

$$
\mathrm{P} S_{F}(D)=10 \log _{10}\left(\frac{P_{t}}{\mathrm{P}_{\mathrm{r}}}\right)=-10 \log _{10}\left[\frac{G_{t} G_{r} C^{2}}{\left(4 \pi D f_{c}\right)^{2}}\right]
$$

Let's imagine the UAV application environment. When UAV data link moves at high speed, the surrounding environment is constantly changing. It will pass through complex landscapes such as urban districts, suburbs, mountains and large buildings, which indicate the change in the characteristics of channel, will not stop in time and the channel will show non-stationary characteristics. Therefore, the path loss coefficient $\delta$ is introduced as the environment changes, which optimized UAV data link channel model [5]. Based on logarithmic distance UAV data link channel model, it is expressed as follows:

$$
\mathrm{P} S_{F D}(D)=-10 \log _{10}\left[\frac{G_{t} G_{r} C^{2}}{\left(4 \pi D_{0} f_{c}\right)^{2}}\right]+10 \delta \log _{10}\left(\frac{D}{D_{0}}\right)
$$

$D_{0}=\left\{\begin{array}{c}1 \sim 100 \mathrm{~m}, D<10 \mathrm{~km} \\ 1000 \mathrm{~m}, D>10 \mathrm{~km}\end{array}\right.$, for different communication environment, $D_{0}$ value changes correspondingly. UAV data link transmission environment path loss index is shown in Table 1 as follows:

TABLE 1 Path loss index table

$\begin{array}{cc}\text { Path loss Coefficient } & \\ \text { Non-obstacle space } & 2 \\ \text { Urban area open area } & 2.7 \sim 3.5 \\ \text { Urban sheltered area } & 3 \sim 5 \\ \text { Visual distance inside building } & 1.6 \sim 1.8 \\ \text { Obstruction in the building } & 4 \sim 6\end{array}$

In the process of UAV data link transmission, a part of the signal will be shaded when the signal transmission path encounters obstacles. This will lead to changes in the average signal, therefore to cause shadow fading phenomenon. When the real channel transmits, the shadow has a random effect and its statistical properties follow a logarithmic normal distribution. Therefore, when the distance is constant and the environment is unchanged, the channel characteristics will change when the data link receiving the platform position changes. Even the distances are the same, each path will also have a different path of decline; UAV data link channel attenuation at this time is closer to the logarithm normal distribution [6]. Among them, order $X_{\sigma}$ indicates that the mean value is 0 ; standard deviation is $\sigma$ Gaussian random variable.

$$
\mathrm{P} S_{F D}(D)=-10 \log _{10}\left[\frac{G_{t} G_{r} C^{2}}{\left(4 \pi D_{0} f_{c}\right)^{2}}\right]+10 \delta \log _{10}\left(\frac{D}{D_{0}}\right)+X_{\sigma}
$$

Logarithm normal path fading model of receiving platform at the same distance D has a different path fading, which varies as the random shadow variable $X_{\sigma}$ changes. 
Factors of transmitting and receiving antennas and the type of environment in the area of propagation have been further studied with the application of Okumura-Hata model to study UAV data link channel.

The path loss in Okumura model can be expressed as follows:

$$
\mathrm{P} S_{O k}(D)=\mathrm{P} S_{F D}(D)+A_{M U}(f, D)-G_{R x}-G_{T x}+G_{A R E A}
$$

$A_{M U}(f, D)$ is moderate fluctuation attenuation factor at frequency f. $G_{R x}$ and $G_{T x}$ are the respective gains of receiving and reflecting of antenna. $G_{A R E A}$ is the propagation environment gain in specific areas. From formula 5 , it is known to spread to the propagation environment, including cities, suburbs and open areas. Taking into account that each path will also have different path fading, therefore, we will add normal distribution characteristics based on the original model. Among them, order $X_{\sigma}$ indicates that the mean value is 0 ; standard deviation is $\sigma$ Gaussian random variable. The specific model is as follows:

(1) The path loss of urban environment is:

$$
L_{C}(D)=69.55+26.16 \log _{10} f_{c}-13.82 \log _{10} h_{R x}-C_{R x}+\left(44.9-6.55 \log _{10} h_{1 x}\right) \log _{10} D+X_{\sigma}
$$

Among them, $C_{R x}$ is correlation coefficient of receiving antenna; $h_{T x}$ is the height of reflecting antenna.

According to UAV data link, the communication coverage is usually quite large. So we use the following formula to get values:

$$
C_{R x}=\left\{\begin{array}{c}
8.29\left(\log _{10}\left(1.54 h_{r x}\right)\right)^{2}-1.1,150 M H z \leq f_{c} \leq 200 M H z \\
3.2\left(\log _{10}\left(11.75 h_{r x}\right)\right)^{2}-4.97,200 M H z \leq f_{c} \leq 1500 M H z
\end{array}\right.
$$

Among them, $h_{R x}$ is the height of reflecting antenna.

(2) The path loss of suburban environment is:

$$
L_{U}(D)=L_{C}(D)-2\left(\log _{10} \frac{f_{c}}{28}\right)^{2}-5.4
$$

(3) The path loss of open area is:

$$
L_{G}(D)=L_{C}(D)-4.78\left(\log _{10} f_{c}\right)^{2}+18.33 \log _{10} f_{c}-40.97
$$

By summarizing the discussion of the channel characteristics of UAV data link, this article determines that the path fading is the main fading factor and establishes the model of channel loss for UAV data link. The simulation and performance analysis of channel loss model for different reference are given as follows.

\section{Channel path loss model analysis}

(1) The effect of antenna gain on the path loss of the UAV data link

UAV data link channel model: UHF channel; using signal frequency: $\mathrm{fc}=900 \mathrm{MHz} ; \sigma=3 \mathrm{db} ; \delta=2$; antenna gain takes different values as shown in the following Table 2:

TABLE 2 Antenna gain table

\begin{tabular}{ccc}
\hline & Transmit antenna gain Receive antenna gain & 2 \\
1 & 1 & 0.5 \\
0.5 & 0.5 & 0.2 \\
\hline
\end{tabular}




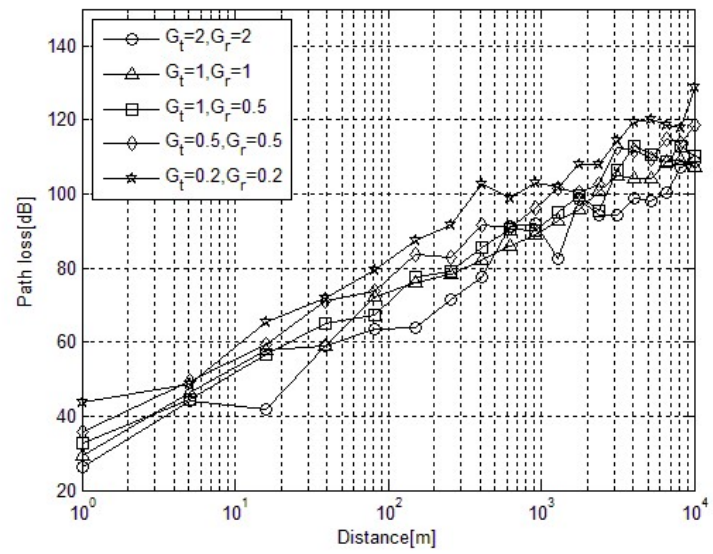

FIGURE.3 Different antenna gains corresponding to channel path transmission attenuation curve

From the simulation result we can see that using the model to simulate different antenna gains is shown in Figure 3. As the receiving and transmitting antennas gain increase, the path attenuation values gradually decrease which accord with the actual measured attenuation.

(2) The influence of signal carrier frequency upon the channel loss of UAV data link

Simulation conditions: the signal carrier frequency values that have been used: $\mathrm{fc}=100 \mathrm{MHz}, 300 \mathrm{MHz}, 800 \mathrm{MHz}$, $1.2 \mathrm{GHz}, 2.0 \mathrm{GHz}$; channel model: ideal UHF channel; antenna gain value remains unchanged in order to satisfy that the transmit antenna gain is $\mathrm{Gt} 3=1$ and receiving antenna gain is $\mathrm{Gr} 3=1$;

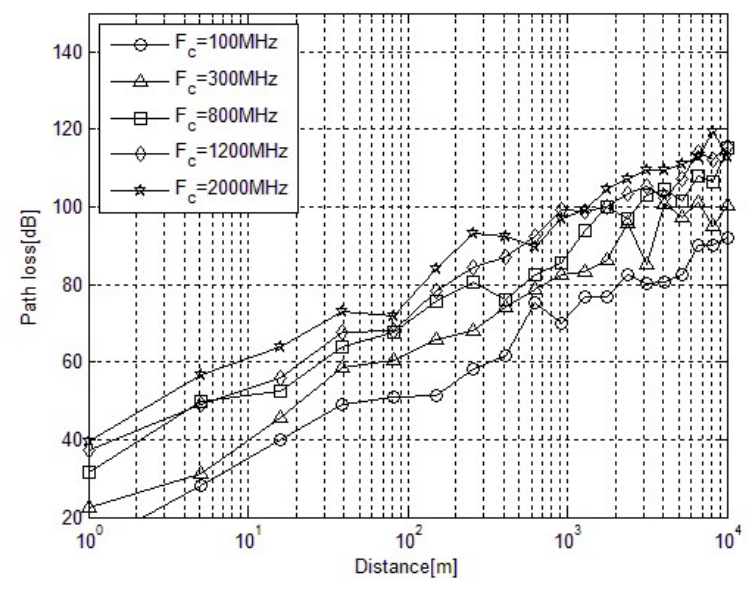

FIGURE.4 Signal carrier frequency affects UAV data link channel path loss curve

The simulation results show that using this simulation model to simulate the different signal carrier frequencies is shown in Figure 4. As the carrier frequency of the received signal increases, the path attenuation tends to be faster, which accords with the actual measured attenuation.

(3) The impact of different location in space upon the channel loss of UAV data link

The frequency of the signal is: $\mathrm{fc}=900 \mathrm{GHz}$; channel model: ideal UHF channel; the antenna gain remains unchanged in order to satisfy that the transmit antenna gain is $\mathrm{Gt} 3=1$ and the receiving antenna gain is $\mathrm{G}$. The impact of path loss parameters is shown in Figure 5 as follows. 


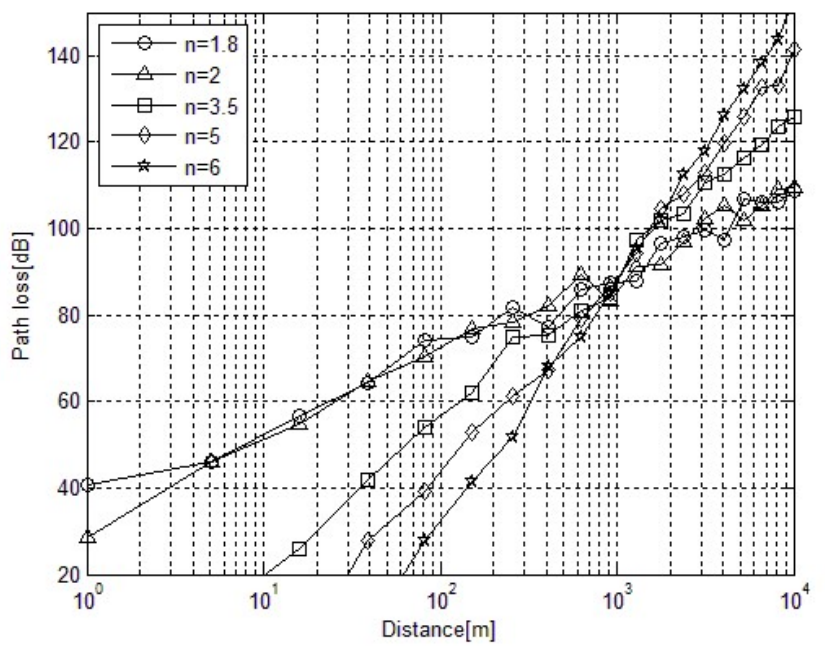

FIGURE.5 Different locations affect UAV data link channel path loss curve

From the simulation results we can see that using this simulation model to simulate different signal carrier frequencies will lead to the following conclusion. Due to the influence of the reference distance, when the distance is greater than the reference distance from the reference distance point 103, as the environmental coefficient increases continuously, the trend of path attenuation will become faster, which is consistent with the trend of actual measured attenuation.

\section{SIMULATION VERIFICATION}

In order to validate the effectiveness of the proposed channel path model in UAV data communication, an area of $200 \mathrm{~km}$ in the desert in suburban area had been selected for this experiment.

(1) Experiment conditions

UAV data link performance is shown in Table 3. Northeastern wind of 3rd level at the speed of $16 \mathrm{~km} / \mathrm{h}$; the weather is fine, the height between aircraft and the ground target is $500 \mathrm{~m}$; the communication distance is $100 \mathrm{~km}$, the antenna frequency coverage is 800 to $1.5 \mathrm{GHz}$; omnidirectional antenna; antenna gain is from $-0.51 \mathrm{~dB}$ to $-0.53 \mathrm{~dB}$; the angle between transceiver antenna and the ground is only $0.57^{\circ}$.

TABLE 3 Antenna gain table

\begin{tabular}{lllllllllll}
\hline Frequency value & $\mathrm{Gr}$ & $\mathrm{Gt}$ & $\mathrm{h}_{\mathrm{Tx}}$ & $\mathrm{hRx}$ & $\mathrm{d} 0$ & $\mathrm{Pt}$ \\
\hline $900 \mathrm{MHz}$ & 0.88 & 0.88 & 500 & 40 & 500 & 47 & \\
\hline
\end{tabular}

(2) Experiment method

(1) Control the UAV flight under stipulated itinerary by controlling the keyboard. The flight altitude increases gradually from $50 \mathrm{~m}$ to $1000 \mathrm{~m}$;

(2) Load the servo antenna, data transmission equipment and image transmission equipment by truck. Control the aircraft to hover, fly back and forth and fly in a circle by controlling the keyboard. The truck rotates in situ (90/180) with constant speed and records the loss value.

(3) Control the aircraft to fly at the altitude of $500 \mathrm{~m}$ according to stipulated itinerary. The truck fixes points of distance at $5 \mathrm{~km}, 40 \mathrm{~km}, 60 \mathrm{~km}, 80 \mathrm{~km}$ and $100 \mathrm{~km}$ along a straight line. The data link will be switched on at fixed time and then switched on during the whole journey. The loss value will be recorded.

(3) Experiment result and theoretical analysis

In UAV data link experiment simulation process, the experiment results are shown in Figure 5 as follows: 


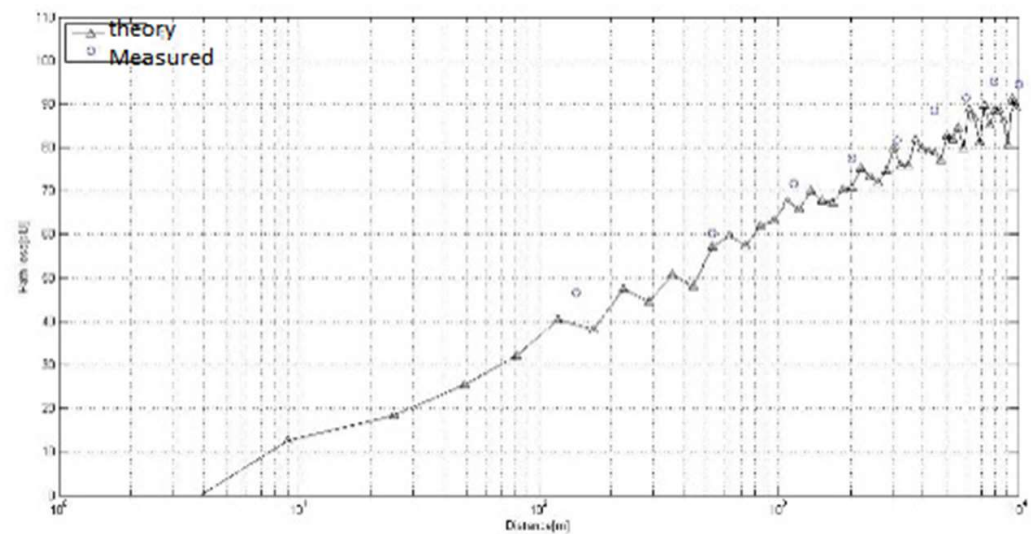

FIGURE.6 Airborne data link communication in different areas Link Fading Curve

Through channel loss model in this article, we can see that the loss calculated in this article satisfies the attenuation trend in the theory and the simulated curve value is less than the real data curve value in Figure 6 . The main reason is that the design model in this article has not considered the small-scale fading factors. Therefore, the curve of the simulation model has $4 \mathrm{~dB} \sim 8 \mathrm{~dB}$ fluctuation compared with the real data curve. The fluctuation values are within a reasonable range. The channel loss model meets the true attenuation trend, which satisfies the theoretical design and research requirements.

\section{CONCLUSION}

This article analyzed the model of data link loss in detail. It established models and stimulated its path loss by the computer. The effects of the signal frequency, the receiving/transmitting antenna gain and the position in space environment on the channel transmission loss have been studied in detail. The factors mentioned above are quantitatively calculated and analyzed by using channel fading model. The reliability of theoretical model is verified by combining actual measured data with theoretical results. This model mainly considers the influence of channel path fading and shadow fading on the link loss. In the actual wireless communication system, the influence of smallscale fading on the channel has been further researched and the attenuation model of the UAV data link communication link has been improved. All these provide an effective theoretical basis for the planning and design of UAV data link communication system.

\section{REFERENCES}

1. YIN Liangliang. A study on some key technologies of UAV flight control [D]. Nanjing: Nanjing University of Aeronautics and Astronautics 2012.

2. F. Prinkney, D.ampel, S.Dipierro.UAV Communication Payload Development, MILCOM Proceeding Volume: 1, 2000, page(s):403-407.

3. Jiang Lei, Wang Yongsheng, Guo Jianxin. UAV-based ultra-width communication chain [J]. Journal of Air Force Engineering University (Natural Science Edition), 2005 (12).

4. El Zein G. Propagation channel modeling for emerging wireless communication systems[C]. Advances in Computational Tools for Engineering Applications, 2009. ACTEA' 09. International Conference on, 2009:457462.

5. Kivinen J.60-GHz wideband radio channel sounder [J]. Instrumentation and Measurement, IEEE Transactions on, 2007, 56(5):1831- 1838.

6. LUTZ E, CYGAN D, DIPPOLD M, et al. The Land Mobile Satellite Communication Channel-Recording, Statistical, and Channel Model [J]. IEEE Trans. Veh. Technol, 1991, 40(2):375-386.

7. GAO Baosheng, ZHU Liangbin. Risk Characteristics of UAVs and Wideband Data Transmission for UAV [J]. Radio Engineering, 2011, 41 (9): 4-6. 